\title{
A comparison study on the flexural strength and compressive strength of four resin- modified luting glass ionomer cements
}

\author{
Yuan $\mathrm{Li}^{\mathrm{a}, \mathrm{b}}$, Hong Lin ${ }^{\mathrm{a}, \mathrm{b}, *}$, Gang Zheng ${ }^{\mathrm{a}, \mathrm{b}}$, Xuehui Zhang ${ }^{\mathrm{a}, \mathrm{b}}$ and Yongxiang Xu $\mathrm{X}^{\mathrm{a}, \mathrm{b}}$ \\ ${ }^{a}$ Department of Dental Materials·Dental Medical Devices Testing Center, Peking University School \\ and Hospital of Stomatology, Beijing, China \\ ${ }^{b}$ National Engineering Laboratory for Digital and Material Technology of Stomatology, Peking \\ University, Beijing, China
}

\begin{abstract}
The purpose of this study is to compare the differences in flexural strength and compressive strength between four resin-modified luting glass ionomer cements that are commonly used in clinics. Furthermore, this study investigates the influence of curing mode on the flexural strength and compressive strength of dual-cured resin-modified glass ionomer cements. Initially, flexural strength and compressive strength test specimens were prepared for RL, NR, GCP, and GCC. The RL group and NR group were cured by the light-curing mode and chemical-curing mode. Five specimens were prepared for each test group, and the flexural strength and compressive strength of each were measured. Data were analyzed by one-way ANOVA with SPSS 13.0. Furthermore, the fracture morphology of the flexural specimens was observed by SEM. The result of the mean flexural strength of each group is as follows: the NR light-cured group $>$ NR chemically-cured group $>$ GCP $>$ RL light-cured group $>$ GCC $>$ RL chemically-cured group. More specifically, the flexural strength of the NR light-cured group $((42.903 \pm 4.242) \mathrm{MPa})$ is significantly higher $(\mathrm{P}<0$. 05) than those of the other groups, and in addition, the flexural strength of the light-curing mode is significantly higher $(\mathrm{P}<0.05)$ than that of both the NR and RL chemically-cured groups. The result of the mean compressive strength of each group is as follows: GCP $>$ NR chemically-cured group $>$ NR lightcured group $>\mathrm{GCC}>\mathrm{RL}$ light-cured group $>\mathrm{RL}$ chemically-cured group. Although the compressive strengths of the NR and GCP groups are higher than those of the GCC and RL groups, there are no significant differences $(\mathrm{P}>0.05)$ between NR and GCP, and no significant differences between GCC and RL. Furthermore, there are no significant differences $(\mathrm{P}>0.05)$ between the two curing modes on NR and RL. From the present study, it can be concluded that NR has superior flexural strength and compressive strength compared to the other three materials. Additionally, the curing mode can affect the flexural strength of dual-cured RMGIC because with the light-curing mode, the flexural strength is higher than with the chemicalcuring mode. Therefore, light curing is an essential procedure when using dual-cured RMGIC in clinics.
\end{abstract}

Keywords: Resin-modified glass ionomer cements, flexural strength, compressive strength, curing mode, light cured and chemical cured

\footnotetext{
* Address for correspondence: Hong Lin, Department of Dental Materials, Peking University School and Hospital of Stomatology, No. 22 South Avenue Zhongguancun, Haidian District, Beijing 100081, China. Tel.: +86 010 82195266; Fax: +8601062164691; E-mail: hong1961in@sina.com.
} 


\section{Introduction}

Previously, $18 \%$ to $20 \%$ resin monomers (HEMA and Bis-GMA and the corresponding activated systems) were added to conventional glass polyalkenoate cements (glass ionomer cement and GIC), which then yielded resin-modified glass ionomer cements (RMGIC) [1]. The resulting RMGIC were demonstrated to be highly advantageous. For example, previous studies have shown that compared to conventional GIC, RMGIC exhibits higher mechanical strength, strong adhesion, lower solubility, and also has fluoride release [2-7]. Therefore, it is apparent that RMGIC is clearly the superior choice, and thus, RMGIC is recommended for clinical use over conventional GIC [7], particularly for such applications as cementation of ceramic or metal prosthesis (metal-based inlays, onlays, crowns, and bridges), high strength (zirconia based) all-ceramic crowns and bridges, and fiber posts and orthodontic attachments. Furthermore, RMGIC can be classified into three types based on the setting characteristics of the polymerizable component: dual-cured, light-cured, and chemically-cured. Most luting cements are classified as either dual-cured or chemically-cured. After the restorations are cemented to the tooth, RMGIC should have the ability to resist the biting force, and such ability can be expressed by compressive strength and flexural strength in the laboratory. Accordingly, the compressive strength and flexural strength of RMGIC are important indicators for the functional evaluation and prerequisites of clinical application. In order to choose the correct curing method and adhesive system, the present study compares the compressive strength and flexural strength of four luting RMGIC. In a clinical situation, light will be partially scattered and absorbed depending on the type, location, and thickness of restoration, and polymerization will usually decrease [8,9]. Therefore, in this study, the influence of the curing mode on the strength of RMGIC is studied, and in addition, the results of the compressive strength and flexural strength of two dual-cured RMGIC, which were respectively cured by light-curing and chemical-curing, are compared.

\section{Experimental method}

\subsection{Materials and equipment}

Table 1 lists all of the materials used in this study.

The following is a list of the equipment employed in this study: mechanical testing machine (INSTRON 3367, USA); EVO-18 SEM (Zeiss, Germany); LED curing light (KERR DEMI, USA); micrometer (Mitutoyo, Japan); water bath (Memmert WNB10, Germany); and a cabinet that was capable of maintaining a temperature of $(37 \pm 1)^{\circ} \mathrm{C}$ and relative humidity of at least $90 \%$ (BLUE PARD LHS-150SC, China).

\subsection{Grouping}

Table 1

Materials used in this study

\begin{tabular}{|c|c|c|c|c|}
\hline Code & Material & Manufacturer & Lot. & Curing mode \\
\hline RL & RelyX ${ }^{\mathrm{TM}}$ luting 2 & 3M ESPE, USA & N490241 & Dual-cured \\
\hline NR & $\begin{array}{l}\text { Nexus }{ }^{\mathrm{TM}} \text { RMGI resin-modified glass ionomer luting } \\
\text { cement }\end{array}$ & $\begin{array}{l}\text { Kerr Corporation, } \\
\text { USA }\end{array}$ & 4882669 & Dual-cured \\
\hline GCP & GC FUJI PLUS resin-modified glass ionomer cement & $\begin{array}{l}\text { GC Corporation, } \\
\text { Japan }\end{array}$ & 130425102881 & $\begin{array}{l}\text { Chemically- } \\
\text { cured }\end{array}$ \\
\hline GCC & $\begin{array}{l}\text { GC FujiCEM resinforced glass ionomer luting } \\
\text { cement }\end{array}$ & $\begin{array}{l}\text { GC Corporation, } \\
\text { Japan }\end{array}$ & 1312061 & $\begin{array}{l}\text { Chemically- } \\
\text { cured }\end{array}$ \\
\hline
\end{tabular}


Specimens were divided into six groups (5 specimens per group): RL light-cured group, RL chemically-cured group, NR light-cured group, NR chemically-cured group, GCP group, and GCC group. (The full material name corresponding to each abbreviation may be found in Table 1)

\subsection{Flexural strength test}

According to ISO 9917-2 [10], flexural strength samples of $2 \mathrm{~mm} \times 2 \mathrm{~mm} \times 25 \mathrm{~mm}$ were prepared. Briefly, the procedure consisted of filling the stainless steel mold with cement immediately after mixing, and the mold was covered with a polyester film and glass plate on both sides. Subsequently, the exit window of the LED curing light was placed as recommended by the manufacturer at the center of the specimen and against the glass plate, followed by light irradiation for the recommended exposure time. Afterwards, the assembly was clamped and placed into a $37^{\circ} \mathrm{C}$ water bath for $15 \mathrm{~min}$. Then, the specimens were removed from the mold, and each specimen was marked at one end so as to indicate the face that was cured first. In addition, if there was any, the excess flash was removed by abrading it with P150 abrasive paper and by avoiding the top and bottom surfaces. After which, the specimens were stored in distilled water at $37^{\circ} \mathrm{C}$ for $24 \mathrm{~h}$. The procedure for the preparation of the chemically-cured group was the same except that these group specimens were not subjected to light activation.

After being stored for $24 \mathrm{~h}$ in $37^{\circ} \mathrm{C}$ water, each specimen was carefully removed from the water bath, and their dimensions were measured to an accuracy of $0.001 \mathrm{~mm}$ using a micrometer at the center of the specimen. Then, the specimens were subjected to flexural strength measurement using a universal testing machine at a crosshead speed of $1.0 \mathrm{~mm} / \mathrm{min}$ until the specimen fractured.

\subsection{Compressive strength test}

According to ISO 9917-1 [11], compressive strength samples of $4 \mathrm{~mm} \times 6 \mathrm{~mm}$ were prepared with a light curing mode and a chemical curing mode separately, as is explained above. The specimens that were cured by the light-curing mode were done so incrementally. After storing the samples in distilled water at $37^{\circ} \mathrm{C}$ for $24 \mathrm{~h}$, the measurements of the specimen dimensions were recorded to an accuracy of $0.001 \mathrm{~mm}$ using the micrometer. Then, the samples were tested for compressive strength using the universal testing machine at a crosshead speed of $1.0 \mathrm{~mm} / \mathrm{min}$ until the specimen fractured.

\subsection{Statistical analysis}

The data of the six groups were analyzed by one-way ANOVA with SPSS 13.0 statistical analysis software $(\alpha=0.05)$.

\subsection{Scanning electron microscope evaluation}

One flexural strength sample in each group was used for scanning electron microscopy (SEM) evaluation after the testing. The SEM micrographs were made at $35 \times$ and $1000 \times$ so as to visually inspect the fracture cross-section morphology.

\section{Results}


Table 2

Flexural strength of different samples in $\mathrm{MPa}$

\begin{tabular}{lcccc}
\hline Group & Max. & Min. & Mean & SD \\
\hline RL light-cured & 23.433 & 16.120 & $18.203^{\mathrm{A}}$ & 2.973 \\
RL chemically-cured & 15.467 & 12.326 & $14.162^{\mathrm{B}}$ & 1.204 \\
NR light-cured & 46.303 & 35.619 & $42.903^{\mathrm{C}}$ & 4.242 \\
NR chemically-cured & 38.977 & 33.787 & $36.448^{\mathrm{D}}$ & 2.121 \\
GCP & 24.780 & 21.932 & $23.577^{\mathrm{E}}$ & 1.214 \\
GCC & 18.843 & 11.209 & $14.722^{\mathrm{B}}$ & 2.813 \\
\hline Note: Groups with different capital letters represent significant difference.
\end{tabular}

Table 3

Compressive strength of different samples in MPa

\begin{tabular}{lcccc}
\hline Group & Max. & Min. & Mean & SD \\
\hline RL light-cured & 89.840 & 80.368 & $86.502^{\mathrm{A}}$ & 3.755 \\
RL chemically-cured & 94.747 & 80.000 & $85.205^{\mathrm{A}}$ & 6.071 \\
NR light-cured & 138.880 & 118.847 & $127.258^{\mathrm{B}}$ & 8.511 \\
NR chemically-cured & 135.704 & 122.616 & $128.501^{\mathrm{B}}$ & 4.933 \\
GCP & 148.071 & 122.761 & $131.729^{\mathrm{B}}$ & 9.875 \\
GCC & 92.730 & 97.717 & $89.829^{\mathrm{A}}$ & 2.311 \\
\hline Note: Groups with different capital letters represent significant difference.
\end{tabular}

\subsection{Test results of flexural strength}

The results of the mean flexural strength of each group is as follows: the NR light-cured group $>$ NR chemically-cured group $>$ GCP $>$ RL light-cured group $>$ GCC $>$ RL chemically-cured group, as is shown in Table 2. The highest mean flexural strength value for the NR light-cured group was 42.903MPa with a standard deviation of $4.242 \mathrm{MPa}$, which is significantly higher $(\mathrm{P}<0.05)$ than that of the other groups. The flexural strength of the light-cured group is significantly higher $(\mathrm{P}<0.05)$ than that of both the NR and RL chemically-cured groups.

\subsection{Test results of compressive strength}

The result of the mean compressive strength of each group is as follows: the GCP group $>$ NR chemically-cured group $>$ NR light-cured group $>$ GCC $>$ RL light-cured group $>$ RL chemicallycured group, as is shown in Table 3. There are no significant differences in the compressive strengths $(\mathrm{P}>0.05)$ between the NR and GCP groups. However, their compressive strengths are higher than those of the GCC and RL groups. The compressive strengths of both the NR and RL group specimens did not significantly differ between the two different curing modes (chemical-curing and light-curing) $(\mathrm{p}>0.05)$.

\subsection{Scanning electron microscopy evaluation}

As observed by both SEM and by the naked eye under the same magnification on the same size field, there were less micro-pores on the fracture cross-section of NR (c, d) and GCC (f) than on the RL (a, b) and GCP (e) samples (Figure 1).

In Figure 1, as observed by both SEM and measured by micrometer, the fracture cross-sectional area of RL (a: $3.285 \mathrm{~mm}^{2}$ ) and NR samples (c: $3.526 \mathrm{~mm}^{2}$ ) with light-cured mode were greater than 
chemically-cured RL (b: $2.970 \mathrm{~mm}^{2}$ ) and NR samples (d: $2.922 \mathrm{~mm}^{2}$ ).

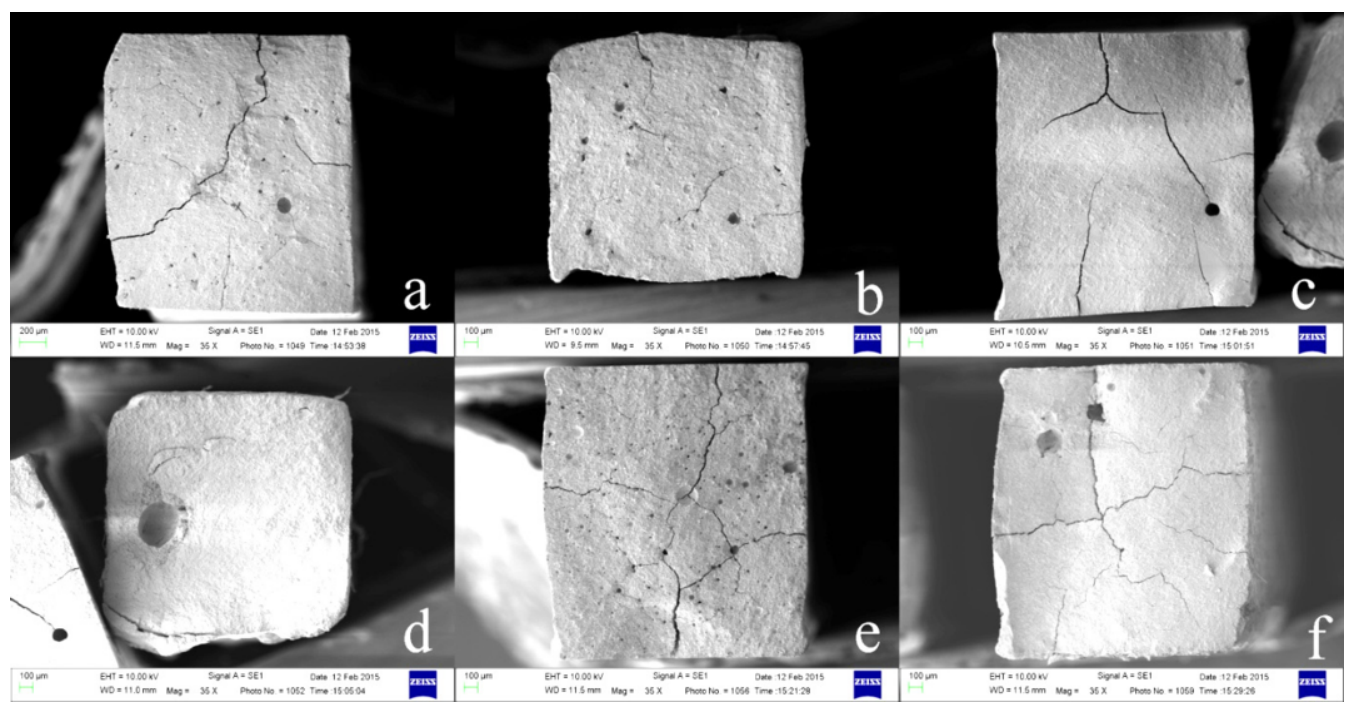

Fig. 1. Morphological evaluation of six flexural fractured cross-section surfaces by SEM (35×): (a) RL light-cured group, (b) RL chemically-cured group, (c) NR light-cured group, (d) NR chemically-cured group, (e) GCP group, and (f) GCC group.

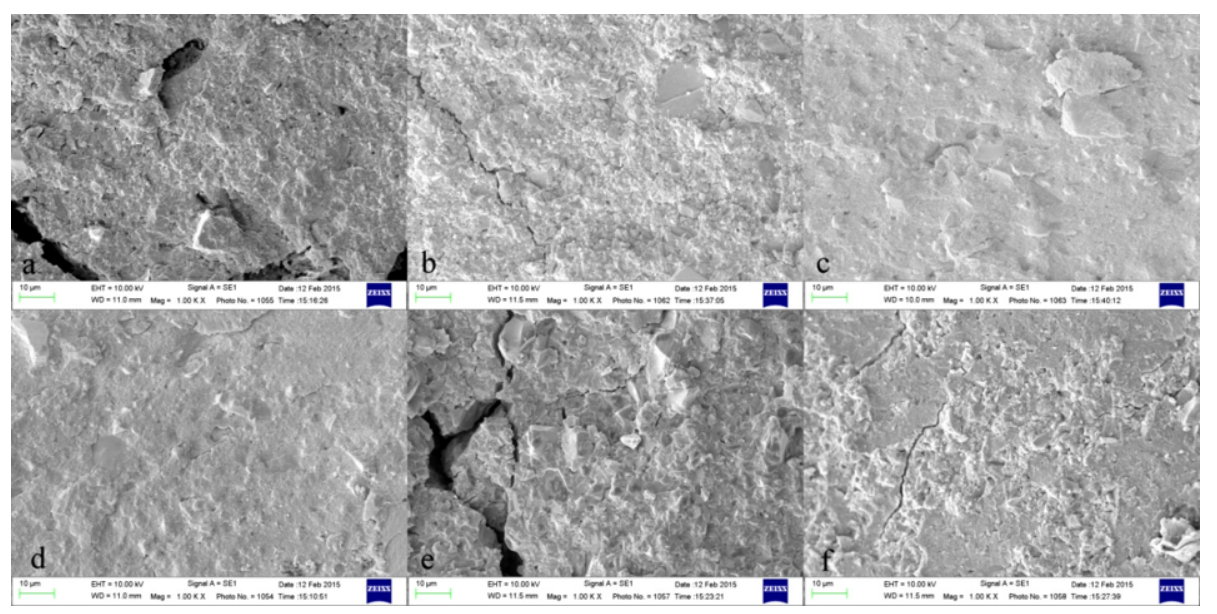

Fig. 2. Morphological evaluation of six flexural fractured cross-section surfaces by SEM (1000×): (a) RL light-cured group, (b) RL chemically-cured group, (c) NR light-cured group, (d) NR chemically-cured group, (e) GCP group, and (f) GCC group.

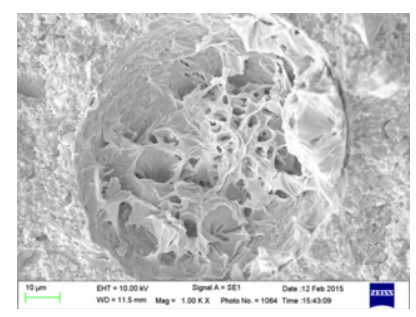

Fig. 3. Morphological evaluation of micro-porous structure on the fracture cross-section of RL observed by SEM (1000×). 
The microstructure of RL (a, b), GCP (e), and GCC (f) was a small granular structure. Compared to the microstructures of the other five groups, NR (c, d) exhibited a compact, flaky structure (Figure 2).

Micro-pores were present in the RL samples, and structures resembling Cicada wings were found in the micro-pores. The structure in the micro-pores differed from the structure surrounding the fracture cross-section (Figure 3).

\section{Discussion}

Under the present study, it can be concluded that NR has a greater flexural strength and compressive strength than the other three materials. Many factors can affect the mechanical properties of RMGIC, such as the chemical composition, microstructure, mixing method, degree of conversion, and the interaction of various factors. Since this study choose four commercial RMGIC, specific chemical compositions and ingredients could not be obtained from manufacturers. Therefore, the microstructure, mixing method, and degree of conversion are discussed in the following.

The microstructure of a material is an important factor of its mechanical strength. The results of the present study showed that the NR group consisted of compact flaky structures (Figures 2(c) and 2(d)), and there was a lack of micro-pores in the samples (Figures 1(c) and 1(d)). The other groups consisted of small granular structures, as deduced from a morphological evaluation by SEM (Figure 2). The differing microstructures influenced the flexural strengths of the four RMGIC; more specifically, the flexural strength of NR was greater than the other materials because the flaky structure of NR has the capacity to resist external force, and this capacity is significantly higher than that of the small granular structure.

Additionally, the mixing method is also an influencing factor on flexural strength. NR was packaged in dual-barrel syringes with single-use automix tips; the two components were completely mixed with a spiral mixer in the barrel syringes, and therefore, no additional air was incorporated via the mixing process. Micro-pores are internal defects of the samples, and they directly affect the mechanical properties of the materials. From the SEM photo (Figures 1(c) and 1(d)) and from visual observation, the NR specimen had significantly less micro-pores on the fracture surface than did the other materials, which is perhaps one reason that NR demonstrated superior mechanical properties. In contrast to NR, the materials of RL were packaged in dual barrel syringes, respectively. A lever was then used to dispense the two components (the base and catalyst) onto a mix pad, where they were mixed with a plastic cement spatula for 20 seconds until a uniform color was achieved. While we sought to avoid incorporating any air bubbles in the mixing process as best as possible, air holes still existed in the RL cured samples (Figures 1(a) and 1(b)). Moreover, GCP consisted of a powder and liquid, which were mixed in accordance with the manufacturer's instructions. Because more air was brought into the material due to hand mixing, more air holes were found inside the cured samples (Figure 1(e)). Thus, the mixing process contributed to disparities in the flexural strength, resulting in NR having a higher strength than either RL or GCP.

Furthermore, the initiators of RMGIC also play an important role in the mechanical properties. Judging from the activated system of the material, both the GCC group and the GCP group were activated by a chemical initiator. In contrast, only the NR group and the RL group were activated by both a photoinitiator and a chemical initiator, which can be determined by the instructions of the product. Dual-cured RMGIC contain two initiator systems: a photoinitiator and a chemical initiator, so the resin monomers can be polymerized by light-curing and chemical-curing processes $[12,13]$. Dualcured RMGIC occur in three stages: after an acid-base reaction at the beginning, the resin monomers 
are polymerized by light-curing and chemical-curing [14]. When the material is irradiated by light, the photoinitiator absorbs energy, and active free radicals are created $[12,15,16]$. Previous studies have shown that the radical polymerization composite resin material quickly creates lots of growth chains in the center and then forms highly cross-linked polymer networks under continuous high-intensity light irradiation. Compared to the light-cured polymerization reaction, the chemically-cured material produces a more linear structure and easily dissolves unreacted monomers, oligomers, and linear polymers [17]. Thus, although the chemically-cured and light-cured processes are independent, light irradiation is a necessary process for the material to achieve an optimal degree of conversion [18-20].

During the specimen preparation, when the chemically-cured samples of RL and NR were removed from the mold, uncured cement was found at the surface of the specimen, and some uncured cement adhesion in the mold. In Figure 1, it showed that the cross-sectional area of the chemically-cured material was smaller than the light-cured material. It suggested that the chemically-cured RMGIC did not polymerize completely, and the extent of polymerization was lower than light-cured material. The cross-linked densities of polymerization from light-curing are often greater than that produced from chemical-curing, and the cross-link densities and degree of polymerization affect the mechanical properties of a polymer-base material that can withstand the load of an external force [21]. Thus, the flexural strength of RL and NR showed a similar behavior, and the flexural strength of the light-cured group was significantly higher than that of the chemically-cured group.

Compressive strength is the ultimate strength to withstand compression stress, and mainly for hard brittle materials, it demonstrates their mechanical behavior during static stretching as reflected in the toughness state of the material [22]. From the results of this study, the curing mode does not significantly affect the compressive strength. Rather, the difference in the compressive strengths of the four luting RMGIC primarily depended upon the composition of the material. In addition, there were no significant differences between the compressive strengths of the two curing modes on dual-cured RGMIC, NR and RL. This is because RMGIC contain polymer components that have a low elastic modulus [23] and greater elastic properties; in addition, the samples that were cured by the chemicalcuring mode become increasingly solid when compressed, except for their fracture. In contrast, the specimens that were cured by the light-curing mode were done so incrementally, and the fracture occurred between layers.

During clinical application, dual-cured RMGIC are intended for permanent cementation, bases, or lining and restoration. When RMGIC are used for crown and bridge cementation, the curing light can be shielded by restorations, thereby causing the light to not be directly exposed to the material as well as not reach the inside of the material. Dentists will light-cure the cement at the interface of restorations and dental tissue and then let the patient occlude for at least five minutes until the setting process is complete. The setting process at the large bonding area of the occlusal surface and the proximal, lingual, and labial surface is achieved by an acid-base reaction combined with chemical polymerization. Based on the experimental data obtained in this study, the light-curing mode could significantly improve the flexural strength of dual-cured RMGIC. Therefore, light-curing is an essential procedure when using dual-cured RMGIC in clinics, especially for the cementing all ceramic crowns and bridges as well as fiber posts and ceramic brackets through which the light can transmit in part, and thus, the capability of resisting the external force of the restorations can be improved by light curing. Furthermore, for metal or metal-based restorations, although the light cannot transmit through the restoration, extending the light exposure time, light intensity and occlude time could improve the effect of cementation.

The flexural strength was frequently used to evaluate dual-cured RMGIC in a laboratory setting, according to ISO 9917-2: 2010. To compare the quality of dual-cured RMGIC, using the light-curing 
mode is adequate. However, for evaluating the function of RMGIC, the worst conditions should be considered for evaluation. For example, if the manufacturer claims that the RMGIC is dual-cured material, an evaluation on the chemical-curing mode should be considered. Not only does this take into account the risk in a worst-case scenario, but it also considers the clinical application from a practical standpoint.

Finally, there was an interesting discovery that structures resembling Cicada wings were found in the micro-pores of RL samples whether the sample was light-cured or chemically-cured (Figure 3). These structures were different from the surrounding particles, and perhaps, the uncured polymer materials exist in micro-pores, and those may be why the flexural strength was weaker than the others. This will be the research focus in the next stage of our study.

\section{Conclusion}

From the present study, it can be concluded that NR has superior flexural strength and compressive strength as compared to the other three materials. Furthermore, the curing mode can affect the flexural strength of dual-cured RMGIC. With the light-curing mode, the flexural strength is higher than with the chemical-curing mode. Therefore, light-curing is an essential procedure when using dual-cured RMGIC in clinics.

\section{Acknowledgment}

This study was supported by the National Natural Science Foundation support program, No. 81470784. All the authors have approved the manuscript, and there are no conflicts of interest to declare.

\section{References}

[1] H. Lin, Resin-modified glass ionomer cements, in: Dental Materials, Peking University Medical Press, Beijing, 2013 , pp. 192-193.

[2] H.J. Prosser, et al., The characterization of glass ionomer cement, Journal of Dentistry 12 (1984), 231.

[3] R.G. Chadwick and M. Woolford, A comparison of the shear bond strengths to a resin composite of two conventional and two resin-modified glass polyalkenoate (ionomer) cements, Journal of Dentistry 21 (1993), 111-116.

[4] D. Kandaswamy, et al., Shear bond strength evaluation of resin composite bonded to glass-ionomer cement using selfetching bonding agents with different $\mathrm{pH}$ in vitro study, Journal of Conservative Dentistry 15 (2012), 27-31.

[5] I.E. Andersson-Wenckert, J.W.V.V. Dijken and P. Hörstedt, Modified class II open sandwich restorations: Evaluation of interfacial adaptation and influence of different restorative techniques, European Journal of Oral Sciences 110 (2002), $270-275$.

[6] R.E. Kerby and L. Knobloch, The relative shear bond strength of visible light-curing and chemically curing glassionomer cement to composite resin, Quintessence International 23 (1992), 641-644.

[7] V. Arora, et al., Comparison of the shear bond strength of RMGIC to a resin composite using different adhesive systems: An in vitro study, Journal of Conservative Dentistry 13 (2010), 80-83.

[8] O.M. El-Mowafy and M.H. Rubo, Influence of composite inlay/onlay thickness on hardening of dual cure resin cements, Journal (Canadian Dental Association) 66 (2000), 147.

[9] C. Prati, S. Chersoni, et al., Effect of air, dentin and resin-based composite thickness on light intensity reduction, American Journal of Dentistry 12 (1999), 231-234.

[10]Dentistry-water-based cements-part 2: Resin-modified cements (ISO 9917-2:2010), available at: http://www.iso.org/iso/home.htm, last accessed Mar. $16^{\text {th }}, 2014$. 
[11]Dentistry-water-based cements-part 1: powder/liquid acid-base cements (ISO 9917-1:2007), available at: http://www.iso.org/iso/home.htm, last accessed Mar. $16^{\text {th }}, 2014$.

[12] M. Bandéca, et al., Changes on degree of conversion of dual-cure luting light-cured with blue LED, Laser Physics 19 (2009), 1050-1055.

[13] M.C.M. Conde, et al., Nanofiller loading level: Influence on selected properties of an adhesive resin, Journal of Dentistry 37 (2009), 331-335.

[14] S. Kasraie, M.Shokripour and M. Safari, Evaluation of micro-shear bond strength of resin modified glass-ionomer to composite resins using various bonding systems, Journal of Conservative Dentistry 16 (2013), 550-554.

[15] A. Tezvergil-Mutluay, L.V.J. Lassila and P.K. Vallittu, Degree of conversion of dual-cure luting resins lightpolymerized through various materials, Acta Odontologica Scandinavica 65 (2007), 201-205.

[16] I. Cekic-Nagas, et al., Influence of polymerization mode on degree of conversion and micropush-out bond strength of resin core systems using different adhesive systems, Dental Materials Journal 27 (2008), 376-385.

[17] J.L. Ferracane, Hygroscopic and hydrolytic effects in dental polymer networks, Dental Materials 22 (2006), 211-222.

[18] M. Bahari, et al., Effect of light intensity on the degree of conversion of dual-cured resin cement at different depths with the use of translucent fiber posts, Journal of Dentistry 11 (2014), 248-255.

[19] S. Flury, et al., Light curing through glass ceramics with a second- and a third- generation LED curing unit: Effect of curing mode on the degree of conversion of dual-curing resin cements, Clinical Oral Investigations 17 (2013), 21272137.

[20]E.D. Souza-Junior, et al., Influence of the curing mode on the degree of conversion of a dual-cured self-adhesive resin luting cement beneath ceramic, Acta Odontologica Scandinavica 71 (2013), 444-448.

[21] A.P.R.V. Pedreira, et al., Microhardness of resin cements in the intraradicular environment: Effects of water storage and softening treatment, Dental Materials 25 (2009), 868-876.

[22] C.Z. Wang, Torsion, bending, compression mechanical properties, in: Performance Materials Science, Beijing Industrial University Press, Beijing, 2001, pp. 39-43.

[23] A. Boruziniat and S. Gharaei, Bond strength between composite resin and resin modified glass ionomer using different adhesive systems and curing techniques, Journal of conservative dentistry 17 (2014), 150-154. 